\title{
Coaching in Hochschule und Wissenschaft
}

\section{Ferdinand Buer}

Für Hochschulen wird in hochindustrialisierten Ländern viel Geld ausgegeben. Da können die Steuerzahler (und andere Finanzierer) schon verlangen, dass hier Hochleistungen vollbracht werden. Dazu werden denn auch mehrfach geprüfte Wissenschaftler/innen eingesetzt. Sie sollen zum einen Forschungsergebnisse produzieren, die international Beachtung finden und vielleicht auch den gesellschaftlichen Fortschritt im eigenen Lande beflügeln. Sie sollen aber zugleich Massen von Studierenden für akademische Berufe qualifizieren und müssen zudem ihren eigenen Nachwuchs heranbilden. Und sie sind gehalten, das alles auch noch selbst angemessen zu managen. Denn dazu stehen ihnen nur schmale Verwaltungen und nur wenige Dienstleistungseinrichtungen zur Seite. Für die Durchführung von Forschungsvorhaben mögen sich die eingestellten Wissenschaftler/ innen im Laufe der Jahre ausreichend gegenseitig qualifiziert haben. Auf Lehre und Management aber sind sie kaum vorbereitet.

Da trifft es viele auf dem falschen Fuß, wenn nun seit einigen Jahren von oben die Hochschulen leistungsfähiger gemacht werden sollen. Das Leitbild ist nun nicht mehr der Professor, der große Werke schafft oder unerhörte Erfindungen macht, oder der Wissenschaftsbeamte, der Wissen anhäuft, verwaltet und weiterreicht. Es ist der Wissenschaftsmanager, der umfangreiche Forschungsprojekte initiiert, möglichst durch Drittmittel finanziert und nützliche Ergebnisse bestimmten Interessengruppen zur Verfügung stellt (Enders und Kaulisch 2005). Dieser Umbau folgt aber in weiten Teilen einer Unternehmenslogik, die ausblendet, dass Hochschulen Expertenorganisationen sind, die zudem gegenüber den Studierenden personenbezogene Dienstleistungen zu erbringen haben. Da muss man sich nicht wundern, dass sich so manche Wissenschaftler/innen fehlgesteuert fühlen und sagen: „Wir sind doch nicht blöd!“ (Knobloch 2010). Diese Reaktanz ist zwar verständlich, jedoch wenig produktiv. In einer Expertenorganisation muss jedes Mitglied nicht nur Experte für sein Fach sein. Er muss auch Experte für die hierarchische wie die kollektive Organisation seiner Tätigkeit in seiner Forschergruppe, seinem Institut, seiner Abteilung, seinem Fachbereich, seiner Hochschule sein.

Online publiziert: 18.01 .2012

(C) VS Verlag für Sozialwissenschaften 2012

Prof. Dr. F. Buer $(\bowtie)$

Alte Schanze 46, 48159 Münster, Deutschland

E-Mail: info@ferdinandbuer.de 
Aber auch die Studienanfänger sind kaum auf ein Ziel orientiertes Studium eingestellt. Auch sie müssen erst einmal lernen, wie man fachspezifisch studiert und wie man sein Studium in der zur Verfügung gestellten Zeit so managt, dass am Ende ein Zeugnis ausgestellt wird, das sich auf dem Markt für höhere Berufe vorzeigen lässt. Wenn nun geringe Lehrkompetenz und geringe Studierkompetenz zusammen kommen - und das bei unterfinanzierten Rahmenbedingungen -, dann ist es verständlich, dass die Ergebnisse nicht sonderlich befriedigen können.

Hier kommt nun das Coaching ins Spiel. Im kollektiven Bewusstsein ist mit ihm ein bestimmtes Versprechen verbunden: Durch Coaching kann eine bestimmte Person ihre Leistungsfähigkeit auf einem bestimmten Gebiet so steigern, dass sie ihre Aufgabe gut, wenn nicht sogar exzellent bewältigen kann. Da es nun bisher an den Hochschulen an Grundausbildungen für Hochschullehrer/innen in Lehre und Management, für Nachwuchswissenschaftler/innen in Wissenschaftsherstellungskompetenz, für Studierende in Lernkompetenz nach wie vor mangelt, muss das alles „on the job“ nachgeholt werden. Das führt an jeder Hochschule zu einer anderen bunten Wiese von Angeboten mit ganz unterschiedlichen Sprösslingen. Coaching ist heutzutage jedoch mit Garantie immer dabei. Eike Hebecker gibt uns in seinem Diskursbeitrag einen kleinen aktuellen Einblick in diese Landschaften.

Schon im Jahre 2009 hat die Zeitschrift OSC diesem Thema ein Schwerpunktheft gewidmet (Buer 2009). Die meisten Beiträge kamen damals von externen Coaches, da in den Hochschulen noch wenig eigene Coachingkompetenz vorhanden war. Einige dieser externen Coaches sind im „Coachingnetz Wissenschaft“ (www.coachingnetzwissenschaft.de) zusammengeschlossen und haben im Jahr 2010 eine eigene Tagung durchgeführt, von der in der OSC berichtet wurde (Klinkhammer et al. 2010). Inzwischen hat sich aber vor allem die hochschulinterne Hochschuldidaktik des Coachings angenommen. So fand im Jahr 2011 an der Technischen Universität Dortmund eine Tagung zum Thema „Coaching als Programm - Beraten (werden) im Feld der Hochschule“ statt, von der in diesem Heft Jutta Wergen, Beatrix Wildt und Johannes Wildt berichten. Somit stehen inzwischen sowohl externe Coaches mit Coachingausbildung und Feldkompetenz zur Verfügung als auch intern ausgebildete Coaches, von denen sich einige im Netz Wissenschaftscoaching (www.wissenschaftscoaching.de) organisiert haben. Die meisten Texte in diesem Heft stammen aus diesem Umfeld. Birgit Szczyrba, Hochschuldidaktikerin und Wissenschaftscoach, sei an dieser Stelle dafür gedankt, viele dieser Beiträge angeregt zu haben.

Den Anfang in diesem Heft macht jedoch eine Externe. Eva-Maria Schumacher gibt uns zunächst einen Überblick über typische Coaching-Anliegen aus dem Bereich Lehre wie dem Bereich Management, exemplarisch dargestellt an der Zielgruppe der Fachhochschullehrenden. Die externen Coaches Bettina Schreyögg und Tanja Nazlic berichten zu Beginn des Praxisteils, wie sie ganz konkret Hochschullehrer/innen im Coaching Kommunikationskompetenz vermitteln, die diese in den verschiedenen Phasen ihrer Hochschulkarriere in verschiedenen Settings dringend benötigen.

Es geht im Coaching aber nicht nur um nachholende Kompetenzvermittlung ,on the job“. Angesichts der gestiegenen Erwartungen an Wissenschaftler/innen und einer erheblichen Vervielfältigung ihrer Rollen, der geringen Aufstiegsmöglichkeiten (viele Habilitierte, aber wenige Lehrstühle) und des rasanten und zudem kaum vorhersehbaren 
Strukturwandels der Wissenschaftslandschaft geraten viele jüngere, aber auch so manche ältere Wissenschaftler/innen in eine Krise. Gerade wenn sie auf vielen Bühnen auftreten und hier auch noch viel Applaus erhalten, überfordern sie sich häufig, merken das aber oft zu spät. Manche kommen sich dann als Hochstapler vor, manchen brennen aus. Die Kommunikationswissenschaftlerin Miriam Meckel hat ihre Erfahrungen mit ihrem Burnout an einem „Brief an mein Leben“ eindrucksvoll beschrieben (Meckel 2010; Buer 2011, S. 77).

Es gibt selbstverständlich auch viele Hochschullehrende, die für diesen Beruf gar nicht geeignet sind. Durch die Nutzung von Seilschaften sind sie auf ihre Posten gekommen. Sie sind nicht nur als Lehrende, sondern allzu oft auch als Forschende hoffnungslos überfordert. Als Beamte auf Lebenszeit sind sie jedoch unkündbar. Hier entspinnen sich häufig Dauerkonflikte zwischen ihnen und ihren Kolleg/innen, aber auch zwischen ihnen und den Instituts- bzw. Hochschulleitungen. In diesen Fällen kann durchaus Konfliktcoaching als begleitende Maßnahme helfen, den Konflikt zumindest einzudämmen. Viele aus dieser Gruppe der Hochschulehrer/innen sind aber ganz einfach nur erschöpft. Sie benötigen ein aufbauendes Coaching, um Erkrankungsprozessen vorzubeugen. Das lässt sich aber wohl nur von externen Coaches mit der nötigen Unabhängigkeit und mit Spezialkompetenzen leisten (Klinkhammer 2011).

Eine Metaanalyse angloamerikanischer Studien zum Burnout unter Lehrenden hat ergeben, dass als wichtigste Ursache für deren psychische Erschöpfung die Überforderung durch die Lehre angesehen werden muss (Watts und Robertson 2011). Das gilt ganz sicher auch für hiesige Verhältnisse. Dabei spielt aber nicht nur die hohe Anzahl an Lehrveranstaltungen, Prüfungen und Begleitung/Bewertung von Abschlussarbeiten eine Rolle. Da für die Vorbereitung, Durchführung und Nachbereitung der Lehre zu oft kaum noch Zeit bleibt, wird diese nur „irgendwie“ durchgeführt. Wenn es also gelänge, Hochschullehrer/innen eine Lehrkompetenz beizubringen, die sie in die Lage versetzt, sachgemäße und adressatenspezifische Lernarrangements anzubieten, die bei den Studierenden Begeisterung und Dankbarkeit auslösen, dann wäre eine wesentliche Ursache für Erschöpfung, Frust und Unlust bei Lehrenden wie bei Lernenden beseitigt. Ja, es könnten sogar Quellen des Glücks freigelegt werden, wenn sich beide Seiten in einem gemeinsamen Erkenntnisstrom wiederfänden (das berühmte „Flow-Phänomen“). Daher stimmt es hoffnungsvoll, dass inzwischen von der Hochschuldidaktik entsprechende Impulse ausgehen.

Frank Linde und Birgit Szczyrba von der Fachhochschule Köln stellen das LehrendenCoaching-Programm ihrer Hochschule vor. Sie beschreiben zunächst, was sie unter Lehrkompetenz verstehen und wie diese in Berufungsverfahren berücksichtigt werden sollte. Neuberufene können dann in einem einjährigen Programm ihre Lehrkompetenz auf den neuesten Stand bringen und durch Coachings unterstützt erproben. Diese Coachings werden durch Hochschuldidaktiker/innen mit Coachingzusatzausbildungen durchgeführt. Ein Programm, durch das Hochschuldidaktiker/innen diese Qualifikation erwerben können, stellen im Anschluss Beatrix Wildt, Birgit Szczyrba und Johannes Wildt, ehemals Leiter des Hochschuldidaktischen Zentrums der TU Dortmund, vor. Durch ein solches Programm wird die Professionalisierung der Hochschuldidaktik erheblich befördert. Coaching wird damit zu einem Standardangebot im Rahmen einer umfassenden Palette, das - systematisch eingesetzt - zur Professionalisierung des gesamten Wissenschaftspersonals beitragen kann. 
Wie der anschließende Beitrag von Matthias Wiemer zeigt, kann es auch sinnvoll sein, bestimmte Studierendengruppen in spezifischen Situationen durch externe wie interne Coaches zu unterstützen. Günstig wäre aber auch, wenn Hochschullehrende ihre eigene Beratungskompetenz durch Coachingqualitäten anreicherten. Dazu bedarf es aber spezifischer Fortbildungen, die nur durch feldkompetente Coachingausbilder durchgeführt werden können.

Soll Coaching ganz offiziell im Rahmen einer Hochschule eingesetzt werden, dann müssen sich nicht nur der Coachee und der Coach einig werden. Es muss auch die Hochschule wissen, was eigentlich wie erreicht werden soll, wenn sie von ihr bezahlte Coaches zur Verfügung stellt. Susanne Gotzen und Jutta Wergen weisen in ihrem Beitrag darauf hin, dass ganz offensichtlich innerhalb einer Hochschule bei denen, die in der Hochschulleitung, in der Personalabteilung, in der Hochschuldidaktik, in verschiedenen Förderprogrammen darüber zu entscheiden haben, keine Übereinstimmung festzustellen ist. Nun kann man meinen, das kommt noch. Coaching ist ja noch neu. Eine Standardisierung sei erst im zweiten Schritt zu erwarten. Gotzen und Wergen sehen aber Hochschulen im Anschluss an den Organisationspsychologen Karl Weick als lose gekoppelte Systeme. Diese sind dadurch gekennzeichnet, dass sich naturwüchsig mehrere Entscheidungszentren herausgebildet haben, zwischen denen Kommunikation nur zufällig und dürftig stattfindet. Das Wunderbare ist jedoch, dass diese vernetzten Systeme durchaus ein langes Leben haben und irgendwie auch wünschbare Leistungen erbringen. Vereinheitlichungsinitiativen sind aber ganz und gar nicht ihre Stärke. Daher ist auch nicht zu erwarten, dass das Coaching an Hochschulen - jeden Falls an großen - jemals standardisiert werden könnte. Meine Anmerkung: Vielleicht sollten Coaching-Beauftragte benannt werden, die die diversen Coachinginitiativen beobachten. Wenn sie dann nach intensiven Konsultationen zum Schluss kommen: „So geht das nicht“", dann sind sie qua Auftrag legitimiert, abratend einzugreifen. Im Konfliktfall muss dann eine höhere Instanz entscheiden. Sie könnten vielleicht auch um Rat gefragt werden, bevor an irgendeiner Stelle in der Hochschule ein neues Coaching-Programm installiert werden soll.

Ein Feld, in dem inzwischen Coachingangebote zum Standard gehören, sind Doktorandenprogramme. Diese werden aber inzwischen nicht nur von den Hochschulen selbst durchgeführt. So bietet etwa das Corporate Center Human Resources der ThyssenKrupp AG ein Programm „Your Innovation“ an, durch das junge Nachwuchswissenschaftler/ innen, die in Kooperation mit einer Universität bei Krupp forschen, personenbezogene Unterstützung erhalten. Die Mitarbeiterinnen dieses Center Kathrin Gimpel und Senta Recktenwald stellen in ihrem Beitrag dieses Programm vor. In diesem Rahmen spielt eine mehrfach stattfindende Kompetenzwerkstatt mit eingebauten kollegialen Coachings eine entscheidende Rolle. Diese Coachings werden angeleitet von Mitgliedern des Netzwerks Wissenschaftscoaching. Auch Stipendiat/innen der Hans-Böckler-Stiftung erhalten Angebote zum kollegialen Coaching im Rahmen eines Projekts „Chancengleichheit in der Begabtenförderung“. Sarah Vespermann und Regina Weber stellen im nachfolgenden Beitrag dar, wie geeignete aktuelle wie ehemalige Stipendiaten zum diversity-sensiblen Coach ausgebildet werden, die dann wiederum nachkommende Stipendiaten coachen können.

Über die Qualität von Wissenschaftscoaching entscheiden also nicht nur Coaches (und deren Berufsverbände), nicht nur Hochschuldidaktik-Zentren, nicht nur Personalabteilungen, Dekanate oder sonstige Hochschulentscheidungszentren. Es entscheiden auch 
Studienförderwerke oder Unternehmen mit Doktorandenprogrammen. So kann Eike Hebecker von der Abteilung Studienförderung der Hans-Böckler-Stiftung mit Recht fragen: „Was heißt und zu welchem Ende coacht man an der Hochschule?“ Man kann die vielen Coaching-Sprösslinge, die hier in den Blick geraten, als Unkraut bezeichnen. Dann müssten sie ausgerottet und die Neuaussaat müsste verboten werden. Aber wer hat in einer lose gekoppelten Organisation die Macht und die Mittel dazu? Man kann sie als Wildwuchs ansehen. Dann müsste es Gärtner mit einheitlichen Standards geben. Die kann man sicher einkaufen. Aber wer will die bezahlen? Und wer will garantieren, dass sie überhaupt auf die Wiese gelassen werden? Man kann die Sprösslinge aber auch einfach wachsen lassen. Es wird immer einige geben, denen ihr baldiges Dahinwelken egal ist. Es wird aber auch einige geben, die werden sich um einige ihrer Lieblingspflanzen so gut kümmern, dass sie durchkommen, ja sogar wachsen und gedeihen. Einige werden sich an diesen Pflanzen erfreuen, werden von ihren süßen Früchten profitieren und möchten keinesfalls auf sie verzichten. Diese sollten sich vernetzen und über Qualität reden und wie sie Qualität erhalten, ausbauen und institutionell absichern können. Und sie sollten zeigen, wieso gerade solche Wiesen mit vielen diversen Coaching-Pflanzen zur Qualitätssicherung von Forschung, Bildung und Management an Hochschulen entscheidend beitragen.

Wer sich über diese Pflanzen jedoch nicht freuen kann und ihre Früchte nicht goutieren will, der muss ja diese bunten Wiesen nicht betreten. Hochschulen bieten viele andere Landschaften von Ackerfeldern über Täler bis zu Hochgebirgen mit ihrem ewigen Eis. Wer sich lieber dort aufhalten will, soll das tun. Das ist sein gutes Recht. Dieses Heft jedenfalls bietet vielfältige Einblicke in bunte Wiesen. Sie laden gerade auch Menschen mit Coachingkompetenzen zum Betreten ein.

\section{Literatur}

Buer, F. (Hrsg.). (2009). Coaching für Wissenschaftler/innen. Themenheft der OSC, 16(2).

Buer, F. (2011). Life-Coaching als Ort der Besinnung. Was Fach- und Führungskräfte dazu bewegen kann, gute Arbeit zu leisten. In C. Schmidt-Lellek \& F. Buer (Hrsg.), Life-Coaching in der Praxis. Wie Coaches umfassend beraten (S. 51-83). Göttingen: Vandenhoeck \& Ruprecht.

Enders, J., \& Kaulisch, M. (2005). Vom Homo Academicus zum Homo Oeconomicus? Die doppelte Kontextualisierung der Forschung und ihre (möglichen) Folgen für die Wissenschaft als Beruf. In M. Pfadenhauer (Hrsg.), Professionelles Handeln (S. 207-220). Wiesbaden: VS Verlag für Sozialwissenschaften.

Klinkhammer, M. (2011). Life-Coaching von Wissenschaftler/innen in der Praxis. In C. SchmidtLellek \& F. Buer (Hrsg.), Life-Coaching in der Praxis. Wie Coaches umfassen beraten (S. 251270). Göttingen: Vandenhoeck \& Ruprecht.

Klinkhammer, M., Pohl, M., \& Hubrath, M. (2010). Tagung des Coachingnetzes Wissenschaft „Coach me, if you can“- Zur Gestaltung von Coachingprogrammen an Hochschulen. OSC, $17(4), 445-448$.

Knobloch, C. (2010). Wir sind doch nicht blöd. Die unternehmerische Hochschule. Münster: Westfälisches Dampfboot.

Meckel, M. (2010). Brief an mein Leben. Erfahrungen mit einem Burnout (4. Aufl.). Reinbek: Rowohlt.

Watts, J., \& Robertson, N. (2011). Burnout in university teaching staff. Educational Research, 53(1), 33-50. 